\title{
Complex Amplitude Filters for Extended Depth of Field
}

\author{
J. Ojeda-Castañeda, E. Yépez-Vidal and E. García-Almanza \\ Electronics Department, University of Guanajuato, Salamanca, 36885, Guanajuato, México
}

Received November 18, 2010; accepted November 23, 2010; published December 31, 2010

\begin{abstract}
We present a complex amplitude transmittance filter that reduces the impact of focus errors on the modulation transfer function (MTF). The new filter combines the use of a cubic phase mask and a Gaussian apodizer, which reduces the oscillations in the MTF. We propose the use of a tendency curve, of several MTFs, for evaluating the departures of the tendency MTF, as a Euclidian distance in hyperspace.
\end{abstract}

\section{Introduction}

Certain nonconventional phase masks, as well as some amplitude apodizers, can reduce the influence of a focus error on the Modulation Transfer Function (MTF) [1][11].

Here we present an optical element that uses both amplitude variations and phase variations, for generating a MTF with a low sensitivity to focus errors. The new filter generates a MTF that varies slowly with the focus error and has reduced oscillations, as compared with previously proposed filters for extending the depth of field.

In section 2 , we show that by combining the use of a mask with a Gaussian apodizer, it is possible to preserve resolution, while reducing the influence of a focus error on the MTF. Furthermore, the Gaussian apodizer reduces the oscillations of the MTF, as compared with previous proposals for extending the depth of field. In section 3, we analyze the decrement in light throughput. In section 4 , we propose the use of a tendency curve, of several MTFs, for evaluating the departures of the tendency MTF, as a Euclidian distance in hyperspace. For the sake of clarity our discussion is 1-D; and we assume that the imaging process has a unit magnification.

\section{Ambiguity function and MTF}

The ambiguity function is a useful mathematical tool for visualizing the impact of a focus error on the MTF [12][15]. The central relationship is the following. We denote the generalized pupil function as

$$
P(\mu ; W)=Q(\mu) \exp \left(i 2 \pi W(\mu / \Omega)^{2}\right) .
$$

*E-mail: jorge_ojedacastaneda@yahoo.com
In Eq. (1) the Greek letter $\mu$ represents the spatial frequency; and $\Omega$ denotes the cut-off spatial frequency of the pupil aperture. The function $\mathrm{Q}(\mu)$ describes the complex amplitude transmittance of the pupil aperture. We use $\mathrm{W}$ as a shorthand notation for representing the 1D version of the Hopkins focus error coefficient, $\mathrm{W}_{2,0}$, in the units of wavelength $\lambda$. That is, $\mathrm{W}=\left(\mathrm{W}_{2,0} / \lambda\right)$.

The optical transfer function, $\mathrm{H}(\mu ; \mathrm{W})$, is a normalized version, $(1 / \mathrm{N})$, of the auto-correlation of $\mathrm{P}(\mu ; \mathrm{W})$

$$
\begin{gathered}
H(\mu ; W)=(1 / N) \int_{-\infty}^{\infty} Q(v+\mu / 2) \quad Q *(v-\mu / 2) \\
\left.\exp \left(i 2 \pi(2 W \mu / \Omega)^{2}\right) v\right) d v
\end{gathered}
$$

The MTF is the modulus of equation (2). The ambiguity function of the complex amplitude transmittance $Q(\mu)$ is

$A_{Q}(\mu, y)=\int_{-\infty}^{\infty} Q(v+\mu / 2) Q^{*}(v-\mu / 2) \exp (i 2 \pi y v) d v$

Consequently, it is convenient to recognize that the ambiguity function, as a 2-D function, contains all the out-of-focus MTFs

$$
\left|A_{Q}\left(\mu, y=2 W \mu / \Omega^{2}\right)\right|=|H(\mu ; W)| .
$$

Equation (3) is useful for relating the results of optical imaging devices with a wavefront design in radar engineering. Moreover, for short time pulses, Eq. (3) helps to visualize the intensity-spectrum evolution through propagation in a first-order dispersive medium. And hence, the ambiguity function helps to design temporal filters that use the degree of temporal coherence of the optical source [16].

For the cubic phase mask, in Ref. [2], the complex amplitude transmittance of the pupil aperture is

$$
Q(\mu)=\exp \left(i 2 \pi a(\mu / \Omega)^{3}\right) \quad \operatorname{rect}(\mu / 2 \Omega) .
$$


In Eq. (4) the letter "a" represents the maximum value of the optical path difference, in units of $\lambda$, which is introduced by the phase mask. By substituting Eq. (4) in Eq. (2), we obtain the modulus of the ambiguity function

$$
\begin{gathered}
\left|A_{Q}(\mu, y)\right|=\mid(1 / 2 \Omega) \int_{-(2 \Omega-|\mu|) / 2}^{(2 \Omega-|\mu|) / 2} \exp \left(i 12 \pi a(\mu / \Omega)(\nu / \Omega)^{2}\right) \\
\exp (i 2 \pi y v) d v \mid .
\end{gathered}
$$

We note that at each value of $\mu$, the integral in Eq. (5) can be expressed in terms of a Fresnel integrals, as was first recognized in radar engineering [17], and later on in optics [18]-[19].

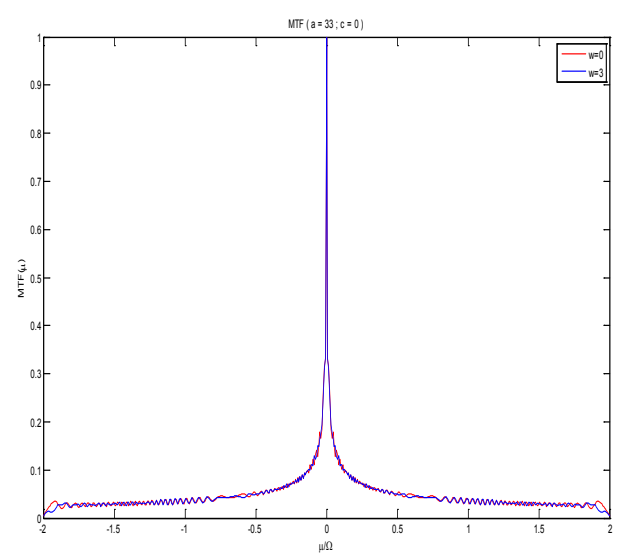

Fig. 1. Typical MTF for the cubic phase mask

In Fig. 1 we show a typical MTF for the cubic phase mask, if the maximum optical path difference is $a=33$. The coefficient of a focus error is $\mathrm{W}=0$ for the curve in a red colour, and $\mathrm{W}=3$ for the curve in a blue colour. It is apparent from Fig. (1) that the MTF does not exhibit zero values in its passband. However, the MTF curve shows oscillations around its tendency line.

Here, for reducing the oscillations around the tendency line, we propose the use of a weak Gaussian filter. Taking into account the presence of the Gaussian apodizer, the new complex amplitude transmittance of the pupil aperture is

$$
G(\mu)=\exp \left(-2 \pi c(\mu / \Omega)^{2}\right) Q(\mu) .
$$

Of course, by changing the attenuation factor "c", one can change the half-width of the Gaussian apodizer. In Figure (2) we show a typical MTF if $a=33, c=0.7, W=0$ for the red colour curve, and $\mathrm{W}=3$ for the blue colour curve.

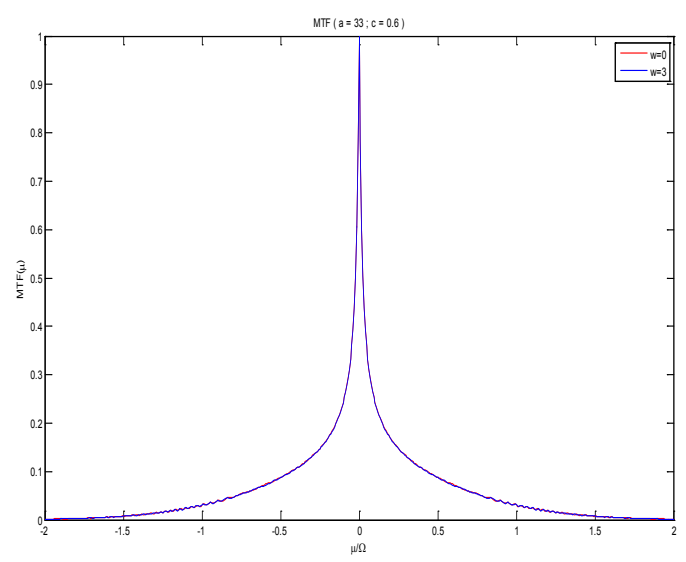

Fig. 2. MTFs for the complex amplitude mask

It is apparent from Fig. 2 that the new MTF preserves low sensitivity to focus errors. But in addition, the new MTF has reduced oscillations around its tendency line. Admittedly, the above results are obtained by sacrificing light throughput, as we discuss next.

\section{Light throughput}

When extending the depth of field there are two main challenges. First, if one wishes to preserve lateral resolution (associated with a full pupil aperture), one avoids the trivial solution of closing down the pupil. Second, if one wishes to preserve light gathering power, then one must use phase-only masks. Here, we use a Gaussian apodizer. Then, we preserve lateral resolution. However, we decrease light that is represented by the integral

$$
\begin{aligned}
T & =(1 / 2 \Omega) \int_{\Omega}^{\Omega}|G(\mu)|^{2} d \mu \\
& =\int_{0}^{1} \exp \left[-4 \pi c(\mu / \Omega)^{2}\right) d(\mu / \Omega) .
\end{aligned}
$$

As discussed in section 4, we need to consider attenuation factors $\mathbb{e} 0.25$. Then, for implementing our proposed complex amplitude filter, light throughput is decreased from unity to 0.49 . In other words, the fnumber is increased by one step.

\section{Tendency curve and Euclidian distance}

Now, we consider a family set of MTFs. Each member of the family is a MTF with a variable focus error W. For instance, we can have a family, of say, 7 MTFs with $\mathrm{W}=0,0.5,1,1.5,2,2.5,3$.

Next, we recognize that we have a large collection of points that are specified by two coordinates: spatial 
frequency, and an MTF value. The collection of points can be thought of as experimental data of a tendency curve. Then, by employing a linear regression technique, one can find a tendency MTF.

The tendency MTF has two useful properties. First, we can use the tendency MTF for evaluating the inverse filter of the family set of MTFs. Second, any departure of a real MTF from the tendency MTF can be associated with an error. The sum of the squared errors is a Euclidian distances in hyperspace.

This Euclidian distance is hyper hypotenuse, which evaluates the departures of a family set of MTFs from the tendency MTF. In other words, the hyper hypotenuse evaluates in a quantitative fashion the statement feature of low sensitivity to a focus error. And consequently, the hyper hypotenuse is useful for assessing the quality of a mask that generates MTFs with low sensitivity to a focus error.

In Fig. 3 we display the hyper hypotenuse (vertical axis) of the proposed complex amplitude mask, as a function the optical path difference "a" in Eq. (4), and the attenuation factor "c" in Eq. (6).

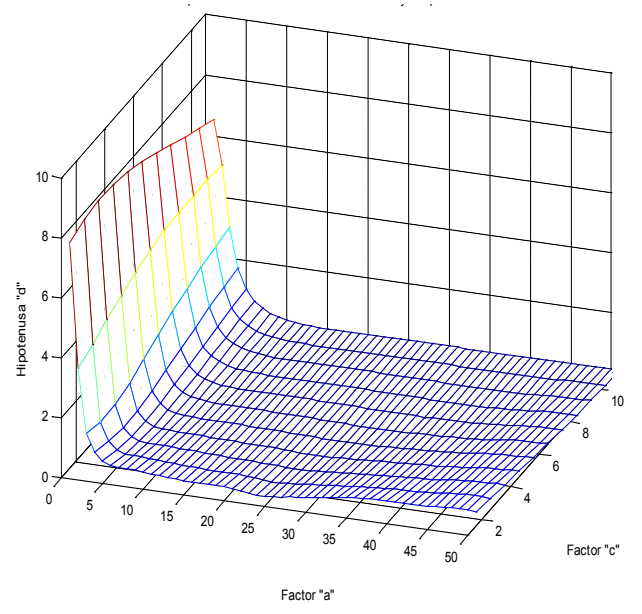

Fig. 3. Hyper hypotenuse of the proposed mask

It is apparent from Fig. 3 that our proposed filter can have low hyper hypotenuse values, if $\mathrm{a} \geq 10$ and $\mathrm{c} \approx 0.25$. In other word, one can use a rather thin version of the phase cubic mask (which is in agreement with the results in Ref. [8]), and a weak Gaussian apodizer. And in this manner, for reducing the oscillations of the MTF one does not have to reduce substantially light throughput.
In conclusions, we have presented a complex amplitude transmittance filter that combines the use of a cubic phase mask and a Gaussian apodizer. This complex amplitude mask reduces the impact of a focus error, as well as the oscillations of the MTF curve.

We have proposed to use several MTFs, with a variable focus error, for generating a family set. By using linear regression techniques, this family set can be used to identify a tendency MTF.

The tendency MTF is useful for identifying an inverse filter for the whole family set of MTFs. Furthermore, any departure of a real MTF from the tendency MTF can be related to a Euclidian hyper hypotenuse, for assessing low sensitivity to a focus error.

From our numerical simulations, we conclude that the hyper hypotenuse reaches a minimum steady state value, if the parameters of the complex filter are set to the following values $\mathrm{a} \geq 10$ and $\mathrm{c} \approx 0.25$.

We are indebted to Erick Ayala for performing initial numerical verifications. We gratefully acknowledge the financial support of PROMEP, SNI and the University of Guanajuato.

\section{References}

[1] J. Ojeda-Castañeda, R. Ramos, A. Noyola-Isgleas, Appl. Opt. 27, 2583 (1988)

[2] E.R. Dowski, Jr., W.T. Cathey, Appl. Opt. 34, 1859 (1995)

[3] W. Chi, N. George, Opt. Lett. 26, 875 (2001).

[4] A. Sauceda, J. Ojeda-Castañeda, Opt. Lett. 29,560 (2004).

[5] S. Mezouari, G. Muyo, A.R. Harvey, Proc. SPIE 5249, 238 (2004).

[6] A. Castro, J. Ojeda-Castañeda, Appl. Opt. 43, 3474 (2004)

[7] J. Ares García, S. Bará, M. Gomez García, Z. Jaroszewicz, A. Kolodziejczyk, K. Petelczyc, Opt. Exp. 16, 18371 (2008).

[8] Y. Takahashi, S. Komatsu, Opt. Lett. 33, 1515 (2008).

[9] J. Ojeda-Castañeda, J.E.A. Landgrave, C.M. Gómez-Sarabia, Appl. Opt., 47, E99 (2008).

[10] P. Mouroulis, Opt. Exp. 16, 12995 (2008).

[11] G. Muyo, A. Singh, M. Andersson, D. Huckridge, A. Wood, A.R. Harvey, Opt. Exp. 17(23), 21118 (2009).

[12] K.-H. Brenner, A.W. Lohmann, Opt. Comm. 77, 89 (1982).

[13] H. Bartelt, J. Ojeda-Castañeda and E.E. Sicre, Appl. Opt. 23, 2693 (1984).

[14] J. Ojeda-Castañeda, L.R. Berriel-Valdos Ramos, E. Montes, Appl. Opt. 27, 790 (1988).

[15] M.E. Testorf, B. Hennelly, J. Ojeda-Castañeda, Phase-Space Optics (New York, McGrawHill, 2010).

[16] J. Ojeda-Castañeda, J. Lancis, C.M. Gómez-Sarabia, V. TorresCompany, Pedro Andrés, J. Opt. Soc. Am. A, 24, 2268 (2007).

[17] C.E. Cook, M. Bernfeld, Radar signals: an introduction to theory and applications (Artech House Inc., Norwood, 1993), p. 120.

[18] G. Muyo, A.R. Harvey, Opt. Lett. 30, 2715 (2005).

[19] M. Somayaji, M.P. Christensen, Appl. Opt. 45, 2911 (2006). 